\title{
A GENERALISATION OF DIRICHLET'S MULTIPLE INTEGRAL
}

\author{
by HENRY JACK
}

(Received 8th May 1964)

1. A previous note (2) showed how the integral of $f\left(\alpha_{1} x_{1}+\alpha_{2} x_{2}+\ldots+\alpha_{n} x_{n}\right)$ over the interior of a simplex could be reduced to a contour integral. The same idea is applied here in Theorems 1 and 2 to give a generalisation of Dirichlet's multiple integral ((1), pp. 169-172). These results are then used in Theorem 3 to reduce an integral over all real $n$-dimensional space to a contour integral. In Theorem 4 an integral over the group of all $3 \times 3$ orthogonal matrices of determinant 1 is reduced to a contour integral. This result can be extended formally to the case of $4 \times 4$ matrices; beyond this it seems difficult to go.

2. In this paragraph theorems 1 and 2 are stated and proved in the case of three variables; the extension to the general case is then obvious.

Theorem 1. Suppose $f(w)=\sum_{n=0}^{\infty} a_{n} w^{n}$ for $|w|<R$, and $\max \{|\alpha|,|\beta|,|\gamma|\}<R$, and $p, q, r$ are positive and $g(w)$ is such that

$$
\iiint_{T} f(\alpha x+\beta y+\gamma z) x^{p-1} y^{q-1} z^{r-1} g(x+y+z) d x d y d z
$$

exists, where $T$ is the region $x \geqq 0, y \geqq 0, z \geqq 0, x+y+z \leqq 1$.

Then the value of (1) is

where

$$
\frac{1}{2 \pi i} \int_{C} \frac{F(w) d w}{(w-\alpha)^{p}(w-\beta)^{q}(w-\gamma)^{r}}
$$

and

$$
F(w)=\Gamma(p) \Gamma(q) \Gamma(r) \sum_{n=0}^{\infty} \frac{G(p+q+r+n)}{\Gamma(p+q+r+n)} n ! a_{n} w^{p+q+r+n-1}
$$

$$
G(p+q+r+n)=\int_{0}^{1} t^{p+q+r+n-1} g(t) d t
$$

and $C$ is the circle $|w|=\rho<R$, enclosing $w=\alpha, \beta, \gamma$.

Proof. By expanding $f(\alpha x+\beta y+\gamma z)$ and using the multinomial theorem on $(\alpha x+\beta y+\gamma z)^{n}$, (1) becomes

$$
\sum_{n=0}^{\infty} n ! a_{n} \sum_{i+j+k=n} \frac{\alpha^{i} \beta^{j} \gamma^{k}}{i ! j ! k !} \iiint_{T} x^{p+i-1} y^{q+j-1} z^{r+k-1} g(x+y+z) d x d y d z
$$


and, by Dirichlet's Integral, this is

$$
\sum_{n=0}^{\infty} n ! a_{n} \sum_{i+j+k=n} \frac{\alpha^{i} \beta^{j} \gamma^{k}}{i ! j ! k !} \frac{\Gamma(p+i) \Gamma(q+j) \Gamma(r+k)}{\Gamma(p+q+r+i+j+k)} \int_{0}^{1} t^{p+q+r+i+j+k-1} g(t) d t .
$$

Using principal values,

so

$$
(1-w)^{-u}=\sum_{s=0}^{\infty} \frac{\Gamma(u+s)}{\Gamma(u) s !} w^{s}
$$

$$
\sum_{i+j+k=n} \frac{\Gamma(p+i) \Gamma(q+j) \Gamma(r+k)}{\Gamma(p) \Gamma(q) \Gamma(r) i ! j ! k !} \alpha^{i} \beta^{i} \gamma^{k}
$$

is the coefficient of $z^{n}$ in the expansion of $(1-\alpha z)^{-p}(1-\beta z)^{-q}(1-\gamma z)^{-r}$, and so $(5)$ is

$$
\frac{1}{2 \pi i} \int_{C^{*}} \frac{1}{(1-\alpha z)^{p}(1-\beta z)^{q}(1-\gamma z)^{r}} \frac{d z}{z^{n+1}},
$$

where $C^{*}$ is the circle

$$
|z|=\rho<R^{*}=\min \left\{|\alpha|^{-1},|\beta|^{-1},|\gamma|^{-1}\right\} .
$$

Now let $w=z^{-1}$ and (4) becomes

$$
\sum_{n=0}^{\infty} n ! a_{n} \frac{G(p+q+r+n)}{\Gamma(p+q+r+n)} \frac{1}{2 \pi i} \int_{C} \frac{\Gamma(p) \Gamma(q) \Gamma(r) w^{p+q+r+n-1}}{(w-\alpha)^{p}(w-\beta)^{q}(w-\gamma)^{r}} d w .
$$

Theorem 2. If $s>0$ and $p+q+r+s=k>1$, and

then

$$
F_{k-1}(w)=\frac{1}{\Gamma(k-1)} \int_{0}^{w} f(t)(w-t)^{k-2} d t
$$

$$
\begin{aligned}
\iiint_{T} f(\alpha x+\beta y+\gamma z) x^{p-1} y^{q-1} z^{r-1}(1-x-y-z)^{s-1} d x d y d z \\
=\frac{\Gamma(p) \Gamma(q) \Gamma(r) \Gamma(s)}{2 \pi i} \int_{C} \frac{F_{k-1}(w) d w}{(w-\alpha)^{p}(w-\beta)^{q}(w-\gamma)^{r} w^{s}} .
\end{aligned}
$$

Proof. By (3), since now $g(w)=(1-w)^{s-1}$,

and so by (2),

$$
G(p+q+r+n)=\int_{0}^{1} t^{p+q+r+n-1}(1-t)^{s-1} d t=\frac{\Gamma(p+q+r+n) \Gamma(s)}{\Gamma(p+q+r+s+n)},
$$

$$
\begin{aligned}
F(w) & =\Gamma(p) \Gamma(q) \Gamma(r) \Gamma(s) \sum_{n=0}^{\infty} \frac{n ! a_{n} w^{p+q+r+n-1}}{\Gamma(k+n)} \\
& =\frac{\Gamma(p) \Gamma(q) \Gamma(r) \Gamma(s)}{w^{s} \Gamma(k-1)} \sum_{n=0}^{\infty} a_{n} w^{k+n-1} \int_{0}^{1} u^{n}(1-u)^{k-2} d u \\
& =\frac{\Gamma(p) \Gamma(q) \Gamma(r) \Gamma(s)}{w^{s} \Gamma(k-1)} \sum_{n=0}^{\infty} a_{n} \int_{0}^{w} t^{n}(w-t)^{k-2} d t
\end{aligned}
$$

which is equivalent to (6). 
GENERALISATION OF DIRICHLET'S MULTIPLE INTEGRAL 235

When $k$ is an integer, $F_{k-1}(w)$ is, to within a polynomial $P_{k-2}(w)$ of degree $k-2$, the $k-1$ times repeated indefinite integral of $f(w)$. But for $\rho \geqq R$,

$$
\int_{|w|=\rho} \frac{P_{k-2}(w) d w}{(w-\alpha)^{p}(w-\beta)^{q}(w-\gamma)^{r} w^{s}}
$$

is, by deformation of contours, a constant with respect to $\rho$; it is also $O\left(\frac{\rho^{k-2} \rho}{\rho^{k}}\right)$ for large $\rho$, so the integral is in fact zero, and the $P_{k-2}(w)$ can be neglected in (7).

3. For the application in the next paragraph only the case $n=3$ of the next result is required. However the form of the result is best seen when working with the general case.

Here and in $\S 4,|M|$ denotes the determinant of the $n \times n$ matrix $M$. Using a dash for the transpose of a matrix, if $x$ is a column vector and

we write $d x=\prod_{r=1}^{n} d x_{r}$.

$$
x^{\prime}=\left\{x_{1}, x_{2}, \ldots, x_{n}\right\},
$$

Theorem 3. Let $S, S_{1}$ be two $n \times n$ symmetric matrices, $S$ being positive definite. Then if $\lambda>0, k>\frac{n}{2}$ and $\mu$ are constants,

$$
\int f\left(\frac{\mu+x^{\prime} S_{1} x}{\lambda+x^{\prime} S x}\right) \frac{d x}{\left(\lambda+x^{\prime} S x\right)^{k}}=\frac{\left\{\Gamma\left(\frac{1}{2}\right)\right\}^{n}}{2 \pi i} \Gamma\left(k-\frac{n}{2}\right) \int_{c} \frac{F_{k-1}(z) d z}{\left|z S-S_{1}\right|^{\frac{1}{2}}(\lambda z-\mu)^{k-\frac{n}{2}}},
$$

the multiple integral, assumed convergent, being taken over all real n-dimensional space and $C$ being a contour inside $|z|=R$, enclosing $z=\mu / \lambda$ and all the roots of $\left|z S-S_{1}\right|=0$.

Proof. Let $S_{2}=\frac{1}{\lambda} S, S_{3}=\frac{1}{\lambda} S_{1}$ and $v=\mu / \lambda$, then the multiple integral in (9) becomes

$$
\frac{1}{\lambda^{k}} \int f\left(\frac{\nu+x^{\prime} S_{3} x}{1+x^{\prime} S_{2} x}\right) \frac{d x}{\left(1+x^{\prime} S_{2} x\right)^{k}}
$$

There is a positive definite symmetric matrix $S_{4}$ such that $S_{2}=S_{4}^{\prime} S_{4}$, so let $\xi=S_{4} x$ and the Jacobian of this transformation is $\frac{\partial(\xi)}{\partial(x)}=\left|S_{4}\right|=\left|S_{2}\right|^{\frac{1}{2}}$ and the multiple integral becomes

$$
\frac{1}{\lambda^{k}\left|S_{2}\right|^{\frac{1}{2}}} \int f\left(\frac{v+\xi^{\prime} S_{4}^{\prime-1} S_{3} S_{4}^{-1} \xi}{1+\xi^{\prime} \xi}\right) \frac{d \xi}{\left(1+\xi^{\prime} \xi\right)^{k}}
$$

Now there is an orthogonal matrix $H$ such that $H^{\prime} S_{4}^{\prime-1} S_{3} S_{4}^{-1} H=\Lambda$, 
where $\Lambda$ is a diagonal matrix, with diagonal elements $\lambda_{1}, \lambda_{2}, \ldots, \lambda_{n}$. Let $\xi=H y$ and the absolute value of the Jacobian $\frac{\partial(\xi)}{\partial(y)}$ is 1 , and (10) becomes

Now

$$
\frac{1}{\lambda^{k}\left|S_{2}\right|^{\frac{1}{2}}} \int f\left(\frac{v+y^{\prime} \Lambda y}{1+y^{\prime} y}\right) \frac{d y}{\left(1+y^{\prime} y\right)^{k}} \text {. }
$$

so let

$$
\begin{aligned}
\frac{v+y^{\prime} \Lambda y}{1+y^{\prime} y} & =\frac{v+\lambda_{1} y_{1}^{2}+\lambda_{2} y_{2}^{2}+\ldots+\lambda_{n} y_{n}^{2}}{1+y_{1}^{2}+y_{2}^{2}+\ldots+y_{n}^{2}} \\
& =v+\frac{\left(\lambda_{1}-v\right) y_{1}^{2}+\left(\lambda_{2}-v\right) y_{2}^{2}+\ldots+\left(\lambda_{n}-v\right) y_{n}^{2}}{1+y_{1}^{2}+y_{2}^{2}+\ldots+y_{n}^{2}}
\end{aligned}
$$

then

$$
z_{r}=\frac{y_{r}^{2}}{1+y_{1}^{2}+\ldots+y_{n}^{2}}, \quad 1 \leqq r \leqq n
$$

and

$$
\frac{\partial\left(z_{1}, z_{2}, \ldots, z_{n}\right)}{\partial\left(y_{1}, y_{2}, \ldots, y_{n}\right)}=\frac{2^{n} y_{1} y_{2} \ldots y_{n}}{\left(1+y_{1}^{2}+\ldots+y_{n}^{2}\right)^{n+1}}
$$

$$
1-z_{1}-z_{2}-\ldots-z_{n}=\frac{1}{1+y_{1}^{2}+\ldots+y_{n}^{2}} \text { and } z_{1} z_{2} \ldots z_{n}=\frac{\left(y_{1} y_{2} \ldots y_{n}\right)^{2}}{\left(1+y_{1}^{2}+\ldots+y_{n}^{2}\right)^{n}} \text {. }
$$

The integral (11) now becomes, since the mapping (12) is $2^{n}$ to 1 ,

$$
\begin{aligned}
\frac{1}{\lambda^{k}\left|S_{2}\right|^{\frac{1}{2}}} \int_{T_{n}} f\left(v+\left(\lambda_{1}-v\right) z_{1}\right. & \left.+\ldots+\left(\lambda_{n}-v\right) z_{n}\right) \\
& \times\left(z_{1} z_{2} \ldots z_{n}\right)^{-\frac{1}{2}}\left(1-z_{1}-z_{2}-\ldots-z_{n}\right)^{k-\frac{n}{2}-1} d z
\end{aligned}
$$

where $T_{n}$ is the region $z_{r} \geqq 0(1 \leqq r \leqq n), z_{1}+z_{2}+\ldots+z_{n} \leqq 1$.

By Theorem 2, (13) is

$$
\frac{\left\{\Gamma\left(\frac{1}{2}\right)\right\}^{n} \Gamma\left(k-\frac{n}{2}\right)}{2 \pi i \dot{\lambda}^{k}\left|S_{2}\right|^{\frac{1}{2}}} \int_{C_{1}} \frac{F_{k-1}(v+w) d w}{\left\{\left(w-\lambda_{1}+v\right)\left(w-\lambda_{2}+v\right) \ldots\left(w-\lambda_{n}+v\right)\right\}^{\frac{1}{2}} w^{k-\frac{n}{2}}} .
$$

Now let $z=v+w$, and since $\left|z I-S_{4}^{-1} S_{3} S_{4}^{-1}\right|=\left(z-\lambda_{1}\right)\left(z-\lambda_{2}\right) \ldots\left(z-\lambda_{n}\right)$, it follows that $\left|z S-S_{1}\right|=\left(z-\lambda_{1}\right)\left(z-\lambda_{2}\right) \ldots\left(z-\lambda_{n}\right)\left|S_{2}\right| \lambda^{n}$, which gives the result.

4. Let $\mathscr{H}_{n}$ denote the compact topological group of all $n \times n$ orthogonal matrices $H$ of determinant +1 , and let $d H$ be the left and right invariant measure on this group. If $f(z)$ is a regular function and $\sigma(M)$ denotes the trace of the matrix $M$, the integral to be evaluated in this paragraph is of the type $\int_{x_{n}} f(\sigma(A H)) d H$, where $A$ is a constant matrix. Now there are $H_{1}, H_{2} \in \mathscr{H}_{n}$ such that $H_{1} A H_{2}=\Lambda$, a diagonal matrix and since $\sigma(A B)=\sigma(B A)$ the integral reduces, by the invariance of the measure, to $\int_{x_{n}} f(\sigma(\Lambda H)) d H$. 
This integral can be transformed into an ordinary multiple integral by using Cayley's parametrisation $H=(I-\Sigma)(I+\Sigma)^{-1}=2(1+\Sigma)^{-1}-I$, where $\Sigma$ is a skew symmetric matrix. It is known ((3), pp. 149-150) that the Jacobian $\partial(H) / \partial(\Sigma)$ of this change of variables is

$$
\frac{2^{\frac{3}{2 n(n-1)}}}{|I+\Sigma|^{n-1}}
$$

Thus the integral becomes

$$
2^{\frac{z}{2 n(n-1)} \int} \int f\left(2 \sigma\left(\Lambda(I+\Sigma)^{-1}\right)-\sigma(\Lambda)\right) \frac{d \Sigma}{|I+\Sigma|^{n-1}},
$$

where $d \Sigma=\prod_{i=1}^{N} d \sigma_{i}$, the $\sigma_{i}$ being the $\frac{1}{2} n(n-1)=N$ elements of $\Sigma$ and the integral being taken over all real $N$-dimensional space.

Theorem 4. If $\Lambda$ has diagonal elements $\alpha, \beta, \gamma$ and $F(z)$ is the indefinite integral of $f(z)$, regular for $|z|<R$, then

$$
\begin{aligned}
\int_{\mathscr{X}_{3}} f(\sigma(\Lambda H)) d H \\
\quad=\frac{v\left(\mathscr{H}_{3}\right)}{2 \pi i} \int_{c} \frac{F(z) d z}{\{(z-\alpha+\beta+\gamma)(z+\alpha-\beta+\gamma)(z+\alpha+\beta-\gamma)(z-\alpha-\beta-\gamma)\}^{\frac{1}{2}}}
\end{aligned}
$$

where $v\left(\mathscr{H}_{3}\right)$ is the Euclidian volume of $\mathscr{H}_{3}$ and $C$ is a contour in $|z|<R$ enclosing all the zeros of the expression in \{\} .

becomes

Proof. If $\Sigma=\left(\begin{array}{rrr}0, & \zeta, & \eta \\ -\zeta, & 0, & \xi \\ -\eta, & -\xi, & 0\end{array}\right)$ the integral on the left hand side of (15)

$2^{2} \int f\left(\frac{(\alpha+\beta+\gamma)+(\alpha-\beta-\gamma) \xi^{2}+(\beta-\gamma-\alpha) \eta^{2}+(\gamma-\alpha-\beta) \zeta^{2}}{1+\xi^{2}+\eta^{2}+\zeta^{2}}\right) \frac{d \xi d \eta d \zeta}{\left(1+\xi^{2}+\eta^{2}+\zeta^{2}\right)^{2}}$

and now Theorem 3 gives the result, since ((3), p. 146), $2^{\frac{2}{2}} \pi^{2}=v\left(\mathscr{H}_{3}\right)$.

\section{REFERENCES}

(1) J. Edwards, A Treatise on the Integral Calculus, Vol. II (London, 1922).

(2) H. JACK, An integral over the interior of a simplex, Proc. Edin. Math. Soc. (2), 13 (1962), 167-171.

(3) F. W. Ponting and H. S. A. Potter, The volume of orthogonal and unitary space, Quart. J. Math. (Oxford), 20 (1949), 146-154.

\section{QUEEN'S COLLEGE}

DUNDEE 